This document is confidential and is proprietary to the American Chemical Society and its authors. Do not copy or disclose without written permission. If you have received this item in error, notify the sender and delete all copies.

\title{
The TACO puzzle: a phase-transition mystery revisited
}

\begin{tabular}{|c|c|}
\hline Journal: & Crystal Growth \& Design \\
\hline Manuscript ID & cg-2018-01461f.R1 \\
\hline Manuscript Type: & Article \\
\hline $\begin{array}{r}\text { Date Submitted by the } \\
\text { Author: }\end{array}$ & $\mathrm{n} / \mathrm{a}$ \\
\hline Complete List of Authors: & $\begin{array}{l}\text { Gavezzotti, Angelo; Universita degli Studi di Milano, Dipartimento di } \\
\text { Chimica } \\
\text { Rizzato, Silvia; Universita degli Studi di Milano, Dipartimento di Chimica } \\
\text { Lo Presti, Leonardo; Università degli Studi di Milano, Department of } \\
\text { Chemistry }\end{array}$ \\
\hline
\end{tabular}




\section{The TACO puzzle: a phase-transition mystery revisited}

Angelo Gavezzotti, ${ }^{a}$ Silvia Rizzato ${ }^{a,}$ and Leonardo Lo Presti ${ }^{a, b, c,{ }^{*}}$

${ }^{a}$ Department of Chemistry, Università degli Studi di Milano, Via Golgi 19, 20133 Milano (Italy)

${ }^{b}$ Centre for Materials Crystallography, Århus University, Langelandsgade 140, DK-8000 Århus (Denmark)

${ }^{c}$ Istituto di Scienze e Tecnologie Molecolari, Italian CNR, Via Golgi 19 I-20133 Milano (Italy)

* To whom correspondence should be addressed: leonardo.lopresti@unimi.it; silvia.rizzato@unimi.it 


\begin{abstract}
The organic salt (5-methyl-1-thia-5-azacyclo-octane-1-oxide) perchlorate (TACO) is known to undergo a single crystal to single crystal phase transition in the 276-298 K $T$ range without change in the external shape of the sample. Despite extensive computational and experimental investigations, no safe conclusions about the transition mechanism could be drawn to now. The two packing patterns are very similar and symmetry is conserved, apart an interchange of cell axes from $P 2_{1} / c(\alpha-\mathrm{TACO}$, low$T)$ to $P 2_{1} / a(\beta-\mathrm{TACO}$, high- $T)$. Yet, the phase transition implies significant conformational rearrangement, coupled with $\sim 180^{\circ}$-wide rotations, of $1 / 2$ of the cations, in conjunction with reorientation of the anions. Here, we analyze the crystal packing of the two phases in terms of pairwise molecule-molecule interaction energies, as derived from the PIXEL approach. Rigid-body molecular reorientations are simulated by solid-state Monte-Carlo calculations, while the likelihood of conformational rearrangements is estimated through gas-phase DFT M06/6-311G(p,d) simulations. We demonstrate that rotational motion of the cations is not hampered by substantial energetic barriers, while ring flip can be described as a two-step process with a main kinetic barrier of $\sim 45 \mathrm{~kJ} \cdot \mathrm{mol}^{-1}$, which might explain the metastable behavior of the $\beta$ phase at low $T$. A possible mechanism of the phase transition is proposed, accounting for the present computational evidences in the context of the former experimental findings.
\end{abstract}




\section{Table of Content Graphic}

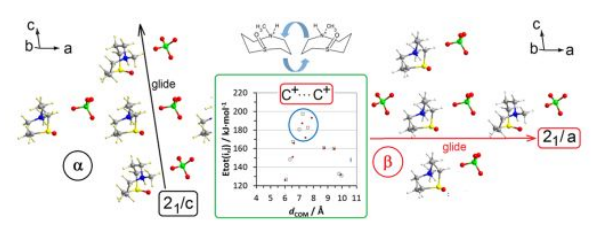

Synopsis TOC: The energy decomposition analysis reveals that the elusive $\alpha \leftrightarrow \beta$ phase transition of TACO should imply a fast rotation of both anions and cations, coupled to a slow boat-chair $\leftrightarrow$ chairboat interconversion of the 8-membred ring. 


\section{Introduction}

Solid-state phase transitions in organic crystals are elusive phenomena occurring over a wide range of conditions and mechanisms, as discussed for example in a recent perspective article ${ }^{1}$ and elsewhere. $^{2-4}$ In some cases the transition occurs without a visible disruption of the mother phase and by an, at least apparently, smooth evolution into the daughter phase. Quite often, and reasonably, preservation of crystalline habit is just apparent and the true mechanism involves an intermediate liquid or liquid-like phase which then re-nucleates into the daughter phase. Analytical methods are several; NMR and IR spectroscopy, ${ }^{5}$ thermochemical analysis ${ }^{6}$ and above all, X-ray diffraction, ${ }^{7,8}$ are common experimental tools for the investigation of transition mechanisms. Simple visual inspection by using a hot-stage microscope is often helpful.9,10 Anyway, cases in which a clear-cut transition path emerges, at a molecular level, are so sparse as to be considered almost chemical curiosities.

We choose for the present case study a particularly puzzling instance that is the evolution of the organic salt (5-methyl-1-thia-5-azacyclo-octane-1-oxide) perchlorate, briefly TACO (Figure 1). Though we are not aware of practical uses or potential applications of this system in materials science or chemistry, it poses problems of fundamental interest in the theory of solid-solid phase transitions. It was first studied by Paul and Go, ${ }^{11}$ revealing two inter-convertible crystal structures, the $\alpha$, low- $T$ phase and the $\beta$, high- $T$ phase. An apparently single crystal-to-single crystal, reversible transition occurs in the 276-298 K range without change in external shape of the sample. In the $\beta$-phase, the $a$ axis is doubled and the $c$ axis is halved, without space group change except for an interchange of axes, easiest seen as a change from $P 2_{1} / c$ to $P 2_{1} / a$. Later, Paul et al. ${ }^{12}$ and Novotny-Bregger ${ }^{13}$ carried out further crystallographic, calorimetric and spectroscopic studies on the transition. Single crystal X-ray diffraction was carried out for $\alpha$ at six temperatures in the range 175 to $288 \mathrm{~K}$ and for $\beta$ at 298,290 , $288 \mathrm{~K}$, and at $193 \mathrm{~K}$ after quenching, but results in the phase transition temperature range were inconclusive. ${ }^{13}$ The boat-chair (BC) form of the ring and intramolecular geometries are constant over temperature and phase change. Thermal parameters of perchlorate oxygens are large, especially in the high- $T$ phase, suggesting some rotational freedom, a common feature in crystals with globular inorganic anions. ${ }^{14}$ The transition energy was estimated by thermal analysis at $2.5 \mathrm{~kJ} \mathrm{~mol}^{-1}$, which implies an estimated $\Delta \mathrm{S}=\Delta \mathrm{H} / \mathrm{T}$ of about $8 \mathrm{~J} \mathrm{~K}^{-1} \mathrm{~mol}^{-1}$ if it is assumed that the transition is a homogeneous process. Inspection of packing diagrams for the two phases shows striking similarities along with subtle differences, but, in spite of extensive investigation, no clues about the existence or 
nature of a stable transition state were found, and no firm conclusions about the molecular transition mechanism could be drawn. Yet, a general consensus exists ${ }^{11-13}$ on the fact that the process should imply a rather easy flip of the azacyclo-octane system between two equivalent boat-chair conformations (Figure 1a), somehow coupled to a supposedly much more difficult, $\sim 180^{\circ}$ wide rotation of the whole cation. Therefore, TACO somehow escapes classical topochemical postulates, which state that solid-state reactions, including structural phase transitions, should proceed with a minimum of atomic and molecular motions. (riferimento?)

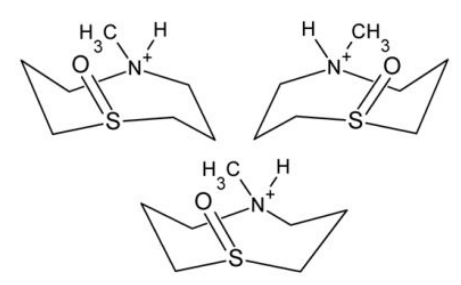

a)

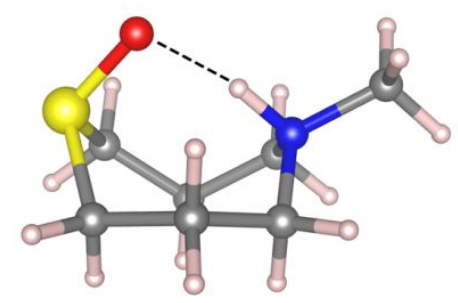

b)

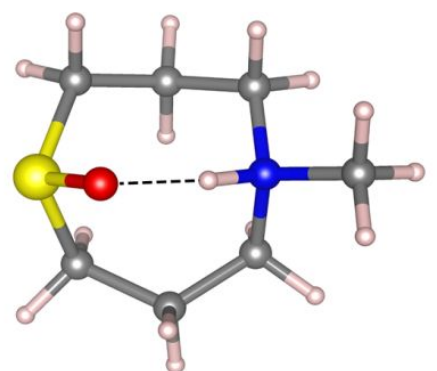

c)

Figure 1. The TACO cation molecule: a) two enantiomers in the boat-chair conformation, and a hypothetical crown conformation; b) and c), two views of the crystalline cation, showing the transannular intramolecular $\mathrm{S}=\mathrm{O} \cdots \mathrm{H}-\mathrm{N}$ hydrogen bond. c) a view down the main inertial axis. The perchlorate anion has a regular tetrahedral shape.

Possible alternative mechanisms were proposed: Parkinson et al. ${ }^{15}$ suggested that the transition could imply a recursive motion of partial dislocations on the (201) (or (102)) crystallographic planes, coupled with an allegedly fast switch between the equivalent boat-chair conformations. Recently, however, Dunitz ${ }^{1}$ pointed out that evidence is lacking that a cooperative shear of alternate planes can occur in ionic crystals. Moreover, it still remains to be proved that the ring flip of TACO is truly not hampered by substantial kinetic barriers.

Theoretical investigations of phase transitions may use packing geometry analysis and static calculations of lattice energies for the involved polymorphic phases, ${ }^{16,17}$ or more complex approaches up to a fully dynamic, temperature-dependent simulation of the process. For organic materials, geometrical packing analysis is elusive, while lattice energy differences between polymorphs are invariably within the limits of accuracy of the theoretical approach, and give no clue as to entropy differences. Dynamic simulation is computationally demanding and can be confidently applied to simple systems for which an exhaustive optimization of the force field has previously been carried 
out. ${ }^{18}$ We propose here an intermediate approach in which the crystal packing of the two phases is analyzed by means of pairwise molecule-molecule cohesion energy contributions, evaluated by the handy and inexpensive PIXEL scheme, which has been shown to give results of comparable accuracy to quantum chemical methods. ${ }^{19}$ As an added bonus, the method allows an energy partitioning into Coulomb-polarization and dispersion terms, which help clarify structures and mechanisms. ${ }^{20}$ This comes as close to the molecular transition level as can be in a non-dynamic approach, revealing the relationships between the local checkerboard of interactions that sustain the transition. Our analysis reveals that, at variance with the usually accepted assumptions, the rotational motion of the cations is not hampered by substantial energetic barriers. On the contrary, our simulations predict that ring flipping acts as a rate determining step, as the pseudorotatory path from any starting boat-chair (BC) to any intermediate crown $(\mathrm{CR})$ conformation implies a kinetic barrier of $\sim 45 \mathrm{~kJ} \cdot \mathrm{mol}^{-1}$. Possible molecular level mechanisms for the phase transition are discussed.

\section{Methods}

Experimental crystal structures of TACO at $T=175,258,278,288 \mathrm{~K}$ (phase $\alpha$ ) and at $T=193$, 288 and $298 \mathrm{~K}$ (phase $\beta$ ) were retrieved from the Cambridge Structural Database. ${ }^{21} \mathrm{C}-\mathrm{H}$ bond lengths were renormalized to the usual neutron-derived estimate ${ }^{22}$ of $1.08 \AA$ through the Retcif module of the CLP/PIXEL program package. ${ }^{23}$ Total molecule-molecule interaction energies $E_{\text {tot }}(i, j)$, as well as lattice energies, $E_{\text {latt}}$, were estimated by means of the PIXEL method, which also allows to partition them into Coulomb $\left(E_{\mathrm{c}}\right)$, polarization $\left(E_{\mathrm{p}}\right)$, dispersion $\left(E_{\mathrm{d}}\right)$ and repulsion $\left(E_{\mathrm{r}}\right)$ contributions. The method is based on interacting molecular charge densities, which were computed at the MP2/6-31G(p,d) level of theory by means of the Gaussian09 program package. ${ }^{24}$ Quantum simulations were performed throughout without imposing any symmetry constraint. Gaussian09 was also employed to estimate the energy barrier for the interconversion between the $\mathrm{BC}$ and $\mathrm{CB}$ conformers. To this end, transition state optimizations were carried out in the gas phase through the Intrinsic Reaction Coordinate (IRC) method $^{25}$ using the Minnesota-class functional M06, ${ }^{26}$ in conjunction with the triple zeta $6-311 G(p, d)$ basis set. The vibrational zero-point correction was applied to all the energies corresponding to refined geometries, including those of transition states.

\section{Results and Discussion}


3.1 Preliminary packing analysis: similarity of the two phases. Essential crystallographic data for TACO are collected in Table 1. The two phases differ in density (Figure S1, ESI) and the thermal expansion coefficients are nearly identical, $\sim 1.6 \cdot 10^{-4} \mathrm{~K}^{-1}$ (phase $\alpha$ ) and $2.2 \cdot 10^{-4} \mathrm{~K}^{-1}$ (phase $\beta$ ). Our theoretical analyses have been conducted using the low- $T \alpha$ phase determination at $278 \mathrm{~K}$ and the high-T $\beta$ phase determination at $298 \mathrm{~K}$, both ordered and defect free, having a comparable degree of $\mathrm{R}$-factor accuracy and thus representing the two phases separated by a good temperature gap and excluding any inter-phase contamination.

Table 1. Crystallographic data for TACO (taken from ref.)

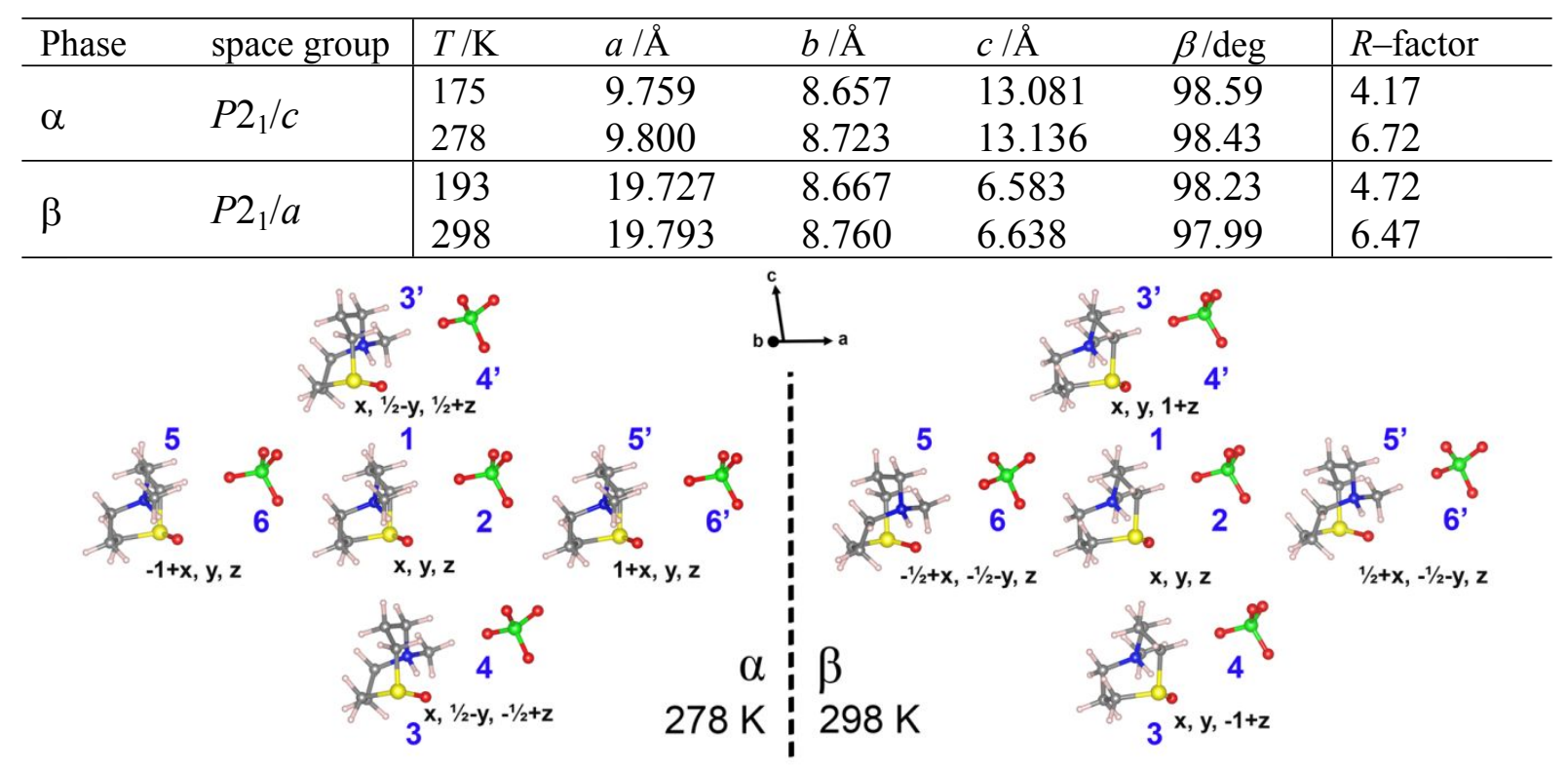

Figure 2. Left: $\alpha$-phase at $278 \mathrm{~K}$ : translation along $a$, horizontal row; glide along $c$, vertical row. Right: $\beta$-phase at 298K: glide along $a$, horizontal row; translation along $c$, vertical row. Symmetry operations and crystallographic reference frames are shown. Numerals serve to unequivocally label pairs of molecules whose contact energies are discussed (see infra); primed numbers refer to translation-related pairs. The $b$ axis is tilted by approximately $\sim 14^{\circ}$ with respect the plane normal of the picture. 


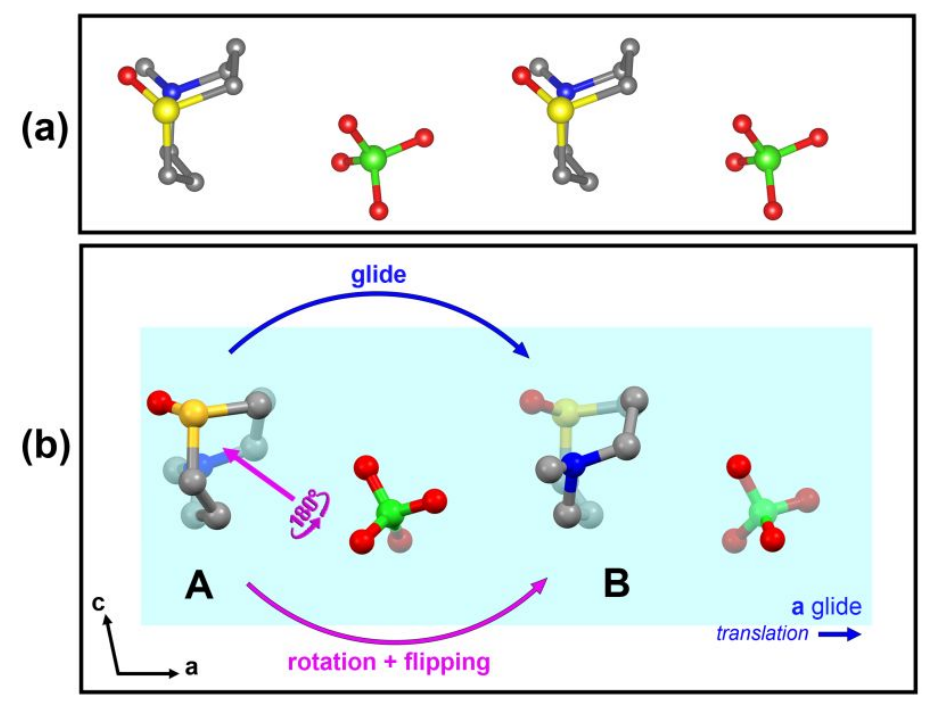

Figure 3. (a) $\alpha$-phase, translation ribbon along $a$. Cation molecules obviously preserve orientation and chirality. (b) $\beta$-phase, the glide reflection along $a$ (x, y, z; $\mathrm{x}+1 / 2,-\mathrm{y}-1 / 2, \mathrm{z})$ (blue arrow) and the "equivalent" rotation-flipping operation (pink arrow) showing that molecule B is transformed with respect to $\mathrm{A}$ by a $180^{\circ}$ rotation around an axis approximately perpendicular to the mean ring plane, along with a boat-chair to chair-boat ring flip (hydrogens omitted for clarity).

A striking similarity between the two crystal structures is apparent on drawing a packing diagram that includes the first few, fundamental interactions in the $a c$ plane of each of them. In the $\alpha-$ phase (Figure 2, left) anion-cation pairs are arranged by translation along the $a$-axis (horizontal) and by glide reflection along the $c$-axis (vertical). In the $\beta$ phase (Figure 2, right) these relationships have switched to glide reflection along the $a$-axis and translation along the $c$-axis, hence the change of space-group setting from $P 2_{1} / c$ to $P 2_{1} / a$.

The distances between centers of mass of the cations are identical (see below), and it is therefore tempting to superimpose the two panels of Figure 2 as naively suggesting a simple rationalization for the transition with cations holding their place in a rigid lattice.

A more in-depth analysis (Figure 3) shows that such a mechanism would require in fact (i) a chirality switch of every second molecule by ring inversion between the two boat-chair forms of the enantiomers, plus (ii) a molecular rotation by $180^{\circ}$ around an axis roughly perpendicular to the mean plane of the ring and (iii) a notable change in the orientation of the anions. Point (iii) may be plausible considering easy rotation of the nearly globular anions, but points (i) and (ii) may involve substantial transition barriers. Each of these points will be analyzed in detail in the following. 
Figure 4 shows another remarkable similarity between the two structures. In both phases the cation coordinates a threesome of anions that contribute the highest stabilization energies (see below). Not surprisingly, these favorable contacts involve a proximity of the negatively charged perchlorate oxygens with the positively charged methylene hydrogen regions, on the opposite side of the S-O group.
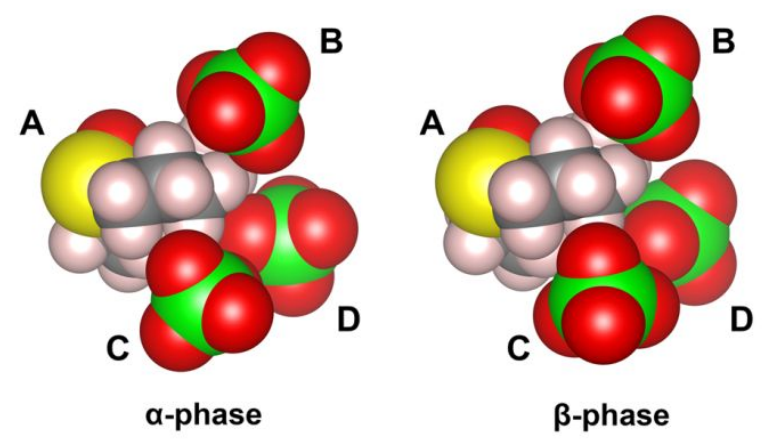

Figure 4. The cation (A) and its three closest-interacting anions (B, C, D). Negative oxygens tend to cluster around positive methylene hydrogen regions in both phases. The A-B contact is almost identical in the two phases.

\subsection{Quantitative energy analysis}

The lattice energies were calculated for both forms at several temperatures by the PIXEL formalism. In that approach the long-range Columbic terms are convergent because lattice summations are carried out on overall neutral units (ion pairs). Phase stability differences should be judged by free energies, which are not within reach of intermolecular energy calculations. However, in an encouraging result, although with reservations about absolute values, the calculation gives the $\beta$-phase as significantly more stable in terms of energies required to pack an ion pair (Table 2). Even a simple atom-atom, point-charge calculation by the AA-CLP scheme gives the same qualitative indication.

Total energies are scarcely sensitive to the temperature of the X-ray determination due to the well known compensation between the parallel increases of dispersion stabilization and overlap repulsion, both short-range terms, as density decreases and molecules come closer together. Further insight into relative stabilities can be gained by an analysis of separate contributions to the noncovalent interaction. As expected in organic salts, ${ }^{13}$ the Coulombic term is largely predominating and accounts alone for the energy difference between phases. For the rest, the above mentioned compensation between polarization-dispersion and repulsion is also at work, in connection with the lower density of the $\beta$-phase. 
Table 2. Total PIXEL lattice energies, $E_{\text {latt }}\left(\mathrm{kJ} \cdot \mathrm{mol}^{-1}\right.$ per cation-anion pair), for the $\alpha$ and $\beta$ phases of TACO at various temperatures. Total energies are decomposed into Coulomb $\left(E_{\mathrm{c}}\right)$, polarization $\left(E_{\mathrm{p}}\right)$, dispersion $\left(E_{\mathrm{d}}\right)$ and repulsion $\left(E_{\mathrm{r}}\right)$ terms $(\operatorname{Section} 2)$.

\begin{tabular}{l|l|lllll}
\hline Phase & $T / \mathrm{K}$ & $E_{\text {latt }}$ & $E_{\mathrm{c}}$ & $E_{\mathrm{p}}$ & $E_{\mathrm{d}}$ & $E_{\mathrm{r}}$ \\
\hline \multirow{4}{*}{$\alpha$} & 175 & -586.4 & -489.0 & -51.0 & -177.6 & 131.0 \\
& 258 & -588.4 & -486.6 & -48.0 & -169.4 & 115.6 \\
& 278 & -587.4 & -483.6 & -47.2 & -167.2 & 110.6 \\
$\beta$ & 288 & -585.8 & -482.2 & -46.4 & -164.4 & 107.0 \\
\hline \multirow{3}{*}{$\beta$} & 193 & -608.8 & -510.0 & -46.0 & -167.8 & 115.0 \\
& 288 & -606.2 & -502.8 & -44.0 & -159.4 & 100.0 \\
& 298 & -603.0 & -500.2 & -41.8 & -155.8 & 94.6 \\
\hline
\end{tabular}

The contact energies, $E^{i, j}$ cont, between pairs of ions in the two crystal structures are calculated in the usual ${ }^{27-30}$ PIXEL setup for all anion-anion, anion-cation and cation-cation pairs. These energies and their separate contribution terms give a close insight into crystal organization and a quantitative estimate of true similarities and differences. If total contact energies were identical by chance, such a misleading indication would be unmasked by a comparison of a set of four numbers. Quite obviously, Coulombic interaction energies between singled-out ions are strongly destabilizing between units of same charge and strongly stabilizing for units of opposite charge; the simple fact that the Coulombic energy between two one-electron charge is $1389.5 / R_{\mathrm{ij}} \mathrm{kJ} \cdot \mathrm{mol}^{-1}$ explains the both the predominance and the long-range nature of these interactions. On the other hand, dispersion energy terms are non-zero only for very first-neighbor interactions, and are one order of magnitude smaller than Coulombic terms.

Table 3. Comparison of center-of-mass distances $\left(d_{\mathrm{COM}}, \AA\right)$ and total molecule-molecule interaction energies $E_{\mathrm{tot}}(i, j)\left(\mathrm{kJ} \cdot \mathrm{mol}^{-1}\right)$ in the $a c$ plane manifolds for the two phases (Figures 2-3). For each molecular pair, the second partner is transformed by the indicated symmetry operation. See Table S1 ESI for full energy partition of interaction energies.

\begin{tabular}{|c|c|c|c|c|c|c|c|}
\hline Species $^{a}$ & Symmetry & Elem. $^{b}$ & Pairs $^{c}$ & Phase & $d_{\mathrm{COM}}$ & $E_{\mathrm{tot}}(i, j)$ & Comp. ${ }^{d}$ \\
\hline \multicolumn{8}{|c|}{ Asymmetric unit } \\
\hline $\mathrm{C}^{+} \cdots \mathrm{A}^{-}$ & $\mathrm{x}, \mathrm{y}, \mathrm{z}$ & $\mathrm{E}$ & $1-2$ & $\alpha$ & 5.083 & -323.6 & VD \\
\hline $\mathrm{C}^{+} \cdots \mathrm{A}^{-}$ & $\mathrm{x}, \mathrm{y}, \mathrm{z}$ & $\mathrm{E}$ & $1-2$ & $\beta$ & 5.387 & -273.4 & VD \\
\hline \multicolumn{8}{|c|}{ c-glide $(\alpha)$ vs. c-translation $(\beta)$} \\
\hline $\mathrm{C}^{+} \cdots \mathrm{C}^{+}$ & $\mathrm{x},-\mathrm{y}-1 / 2, \mathrm{z} \pm 1 / 2$ & G & $1-3,1-3^{\prime}$ & $\alpha$ & 6.571 & 167.2 & ID \\
\hline $\mathrm{C}^{+} \cdots \mathrm{C}^{+}$ & $\mathrm{x}, \mathrm{y}, \mathrm{z} \pm 1$ & $\mathrm{~T}$ & $1-3,1-3^{\prime}$ & $\beta$ & 6.638 & 165.9 & ID \\
\hline $\mathrm{C}^{+} \cdots \mathrm{A}^{-}$ & $\mathrm{x},-\mathrm{y}-1 / 2, \mathrm{z}-1 / 2$ & G & $1-4$ & $\alpha$ & 6.091 & -258.6 & VD \\
\hline
\end{tabular}




\begin{tabular}{|c|c|c|c|c|c|c|c|}
\hline $\mathrm{C}^{+} \cdots \mathrm{A}^{-}$ & $x,-y-1 / 2, z+1 / 2$ & G & $2-3^{\prime}$ & $\alpha$ & Id. & & VD \\
\hline $\mathrm{C}^{+} \cdots \mathrm{A}^{-}$ & $\mathrm{x}, \mathrm{y}, \mathrm{z} \pm 1$ & $\mathrm{~T}$ & $2-3^{\prime}, 1-4$ & $\beta$ & 7.707 & -151.6 & VD \\
\hline $\mathrm{C}^{+} \cdots \mathrm{A}^{-}$ & $x,-y-1 / 2, z+1 / 2$ & $\mathrm{G}$ & $1-4^{\prime}$ & $\alpha$ & 10.040 & -149.4 & $\mathrm{D}$ \\
\hline $\mathrm{C}^{+} \cdots \mathrm{A}^{-}$ & $x,-y-1 / 2, z-1 / 2$ & $\mathrm{G}$ & $2-3$ & $\beta$ & Id & & $\mathrm{D}$ \\
\hline $\mathrm{C}^{+\cdots} \mathrm{A}^{-}$ & $\mathrm{x}, \mathrm{y}, \mathrm{z} \pm 1$ & $\mathrm{~T}$ & $1-4^{\prime}, 2-3$ & $\beta$ & 9.315 & -161.1 & $\mathrm{D}$ \\
\hline $\mathrm{A}^{-} \cdot \cdots \mathrm{A}^{-}$ & $\mathrm{x}, \mathrm{y}-1 / 2, \mathrm{z} \pm 1 / 2$ & G & $2-4,2-4^{\prime}$ & $\alpha$ & 6.574 & 206.4 & ID \\
\hline $\mathrm{A}^{-\cdots} \mathrm{A}^{-}$ & $\mathrm{x}, \mathrm{y}, \mathrm{z} \pm 1$ & $\mathrm{~T}$ & $2-4^{\prime}, 2-4$ & $\beta$ & 6.638 & 204.9 & ID \\
\hline \multicolumn{8}{|c|}{ a-translation $(\alpha)$ vs. a-glide $(\beta)$} \\
\hline \multirow{3}{*}{$\begin{array}{l}\mathrm{C}^{+} \cdots \mathrm{C}^{+} \\
\mathrm{C}^{+} \cdots \mathrm{C}^{+}\end{array}$} & $\mathrm{x} \pm 1, \mathrm{y}, \mathrm{z}$ & $\mathrm{T}$ & $1-5,1-5^{\prime}$ & $\alpha$ & 9.800 & 132.8 & ID \\
\hline & $\mathrm{x} \pm 1 / 2, \quad-\mathrm{y}-1 / 2$ & G & $1-5,1-5$ & $\beta$ & & & \\
\hline & $\mathrm{z}$ & & & & 9.904 & 131.8 & ID \\
\hline $\mathrm{C}^{+} \cdots \mathrm{A}^{-}$ & $\mathrm{x} \pm 1, \mathrm{y}, \mathrm{z}$ & $\mathrm{T}$ & $1-6,2-5^{\prime}$ & $\alpha$ & 5.329 & -271.0 & VD \\
\hline $\mathrm{C}^{+} \cdots \mathrm{A}^{-}$ & $x-1 / 2,-y-1 / 2, z$ & G & $1-6$ & $\beta$ & 5.151 & -317.1 & VD \\
\hline $\mathrm{C}^{+\cdots} \mathrm{A}^{-}$ & $\begin{array}{l}x+1 / 2, \quad-y-1 / 2, \\
z\end{array}$ & G & $2-5^{\prime}$ & $\beta$ & Id. & & VD \\
\hline $\mathrm{A}^{-} \cdot \cdots \mathrm{A}^{-}$ & $\mathrm{x} \pm 1, \mathrm{y}, \mathrm{z}$ & $\mathrm{T}$ & $2-6,2-6$ & $\alpha$ & 9.800 & 140.9 & ID \\
\hline $\mathrm{A}^{-\cdots} \mathrm{A}^{-}$ & $x \pm 1 / 2,-y-1 / 2, z$ & G & $2-6,2-6$ & $\beta$ & 9.932 & 138.7 & ID \\
\hline \multicolumn{8}{|c|}{ Cation-Cation interactions along $b$} \\
\hline $\mathrm{C}^{+} \cdots \mathrm{C}^{+}$ & $\mathrm{x}, \mathrm{y}+1, \mathrm{z}$ & $\mathrm{T}$ & - & $\alpha$ & 8.723 & 160.9 & ID \\
\hline $\mathrm{C}^{+} \cdots \mathrm{C}^{+}$ & $\mathrm{x}, \mathrm{y}+1, \mathrm{z}$ & $\mathrm{T}$ & - & $\beta$ & 8.760 & 160.4 & ID \\
\hline $\mathrm{C}^{+} \cdots \mathrm{C}^{+}$ & $-\mathrm{x}, 1 / 2+\mathrm{y}, 1 / 2-\mathrm{z}$ & G & - & $\alpha$ & 9.434 & 160.2 & ID \\
\hline $\mathrm{C}^{+} \cdot \cdots \mathrm{C}^{+}$ & $-x-1 / 2,1 / 2+y,-z$ & G & - & $\beta$ & 9.458 & 159.7 & ID \\
\hline
\end{tabular}

${ }^{a} \mathrm{C}^{+}$: Cation; $\mathrm{A}^{-}$: Anion.

${ }^{b}$ Symmetry element involved. E: identity; G: glide; T: translation.

${ }^{c}$ See Figure 2 for pair numbering. A "-" means that the pair is not shown in Figure 2.

${ }^{d}$ Qualitative descriptor for comparing the total interaction energies. VD: very different; D: Different; ID: Identical.

We first analyze the surprising similarity of the layer structures shown in Figures 3-4. Cationcation and anion-anion R's and $E^{i, j}$ 's (Table 3 ) along the glide reflection and along the translation displacements in the $a c$ plane are identical after the interchange in the two phases, in spite of the change of symmetry, chirality and orientation. However, cation-anion arrangements are quite different. Thus, the ac-plane manifolds that appear very nearly identical in the two structures on visual examination, or even judging from the similarity of center-of-mass separations, in fact conceal a number of very different intermolecular contact energies. This result once again underscores the need for quantitative energetic considerations in judging packing similarities or differences.

Out of the $a c$ plane the two structures also present a striking similarity (see the last entries in Table 3), with identical cation-cation translations along $b$ and screw-related pairs also along $b$ in $P 2_{1} / a$ and $P 2_{1} / c$. 
Table 4 shows the contact energies of the three most stabilizing cation-anion contact in the two phases, as shown in Figure 5. The A-B contact is nearly identical in the two phases but the other two show only a broad geometrical and energetic similarity. Negative oxygens tend to cluster around positive methylene hydrogen regions in both phases; an overall view of the comparison between the two phases is provided by a complete plot of molecule--molecule contact energies against center-ofmass distance, including all symmetry-related pairs.

Table 4. Comparison of center-of-mass distances $\left(d_{\mathrm{COM}}, \AA\right)$ and molecule-molecule interaction energies $\left(\mathrm{kJ} \cdot \mathrm{mol}^{-1}\right)$, partitioned into Coulomb $\left(E_{\mathrm{c}}\right)$, polarization $\left(E_{\mathrm{p}}\right)$, dispersion $\left(E_{\mathrm{d}}\right)$ and repulsion $\left(E_{\mathrm{r}}\right)$ terms, in the closest cation-anion contacts (see Figure 4).

\begin{tabular}{l|lll|lllll|l}
\hline Pair & Symmetry & Elem. $^{a}$ & Phase & $d_{\text {COM }}$ & $E_{\mathrm{c}}$ & $E_{\mathrm{p}}$ & $E_{\mathrm{d}}$ & $E_{\mathrm{r}}$ & $E_{\text {tot }}$ \\
\hline A-B & $-\mathrm{x},-\mathrm{y},-\mathrm{z}$ & $\mathrm{I}$ & $\alpha$ & 5.278 & -273.8 & -28.7 & -17.2 & 11.2 & -308.5 \\
A-B & $-\mathrm{x},-\mathrm{y},-1-\mathrm{z}$ & $\mathrm{I}$ & $\beta$ & 5.196 & -280.4 & -31.6 & -18.2 & 13.5 & -316.6 \\
A-C & $\mathrm{x}, \mathrm{y}, \mathrm{z}$ & $\mathrm{E}$ & $\alpha$ & 5.083 & -287.9 & -30.6 & -16.0 & 10.9 & -323.6 \\
A-C & $\mathrm{x}-1 / 2,-\mathrm{y}-1 / 2, \mathrm{z}$ & $\mathrm{G}$ & $\beta$ & 5.151 & -282.2 & -29.8 & -14.6 & 9.5 & -317.1 \\
A-D & $-\mathrm{x}, \mathrm{y}+1 / 2,-\mathrm{z}+1 / 2$ & $\mathrm{~S}$ & $\alpha$ & 6.053 & -274.8 & -26.2 & -13.0 & 13.0 & -300.9 \\
A-D & $-\mathrm{x},-\mathrm{y},-\mathrm{z}$ & $\mathrm{I}$ & $\beta$ & 5.699 & -284.6 & -27.6 & -15.4 & 9.1 & -318.4 \\
\hline
\end{tabular}

${ }^{a}$ Symmetry element involved. I: inversion; E: identity; G: glide; S: screw axis.

Figure 5 shows these distance/energy landscapes, summarizing in a graphical manner, among others, the data collected in Tables $2-3$. The cation-cation pattern shows the previously mentioned similarities but also points out a region of dissimilarity in the 7-8 $\AA$ range. Interestingly, a large shape effect appears, because the electron density of the cation is far from a spherical distribution: in fact, the short-distance range is less repulsive than the medium-distance range, thanks to partially stabilizing effects due to the distribution of positive nuclear and negative electron charges. The two closest anionanion contacts are almost identical in the two phases and then the pattern merges into a distribution strictly coincident with Coulomb's law for a spherical ion. ${ }^{14}$ 


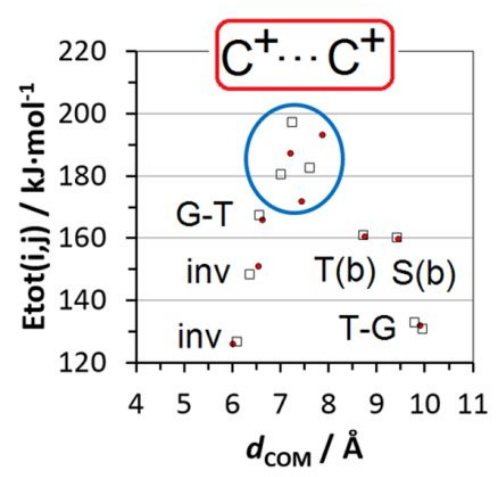

(a)

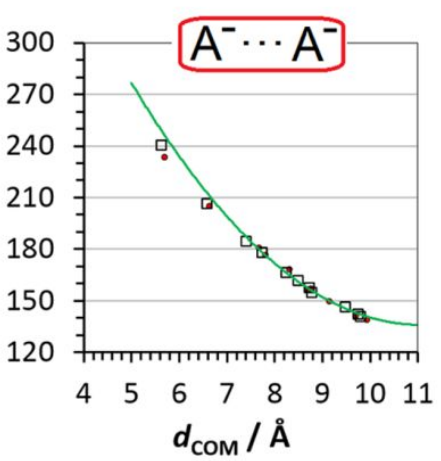

(b)

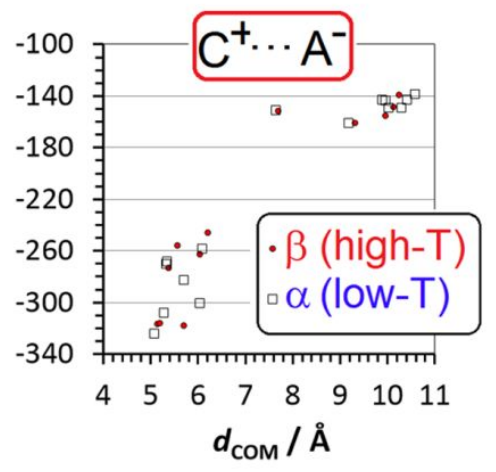

(c)

Figure 5. (a) Distance-energy pattern for the cation-cation contacts in the two phases. $d_{\mathrm{COM}}$ is the centre-of-mass distance. Identical pairs: two inversion-related (inv) plus one $b$-translation (T(b)) and one screw pair $(\mathrm{S}(\mathrm{b}))$. G-T and $\mathrm{T}-\mathrm{G}$ are the pairs interchanged between translation and glide in the $a c$ plane. The blue circle encloses pairs where $d_{\mathrm{COM}}$ changes by about $0.3 \AA$ and $E_{\mathrm{tot}}(i, j)$ by about $10 \mathrm{~kJ}$ mol $^{-1}$ on changing phase. (b) Same as (a), for the anion-anion contacts. The full green line follows the Coulomb law for a couple of ions with identical charges, as a function of $d_{\mathrm{COM}}$. (b) Same as (a), for the cation-anion contacts.

The anion-cation pattern (Figure 5c) shows significant structural reshuffling in the closest-neighbor 5$6 \AA$ distance range, then a 6-10 $\AA$ distance region almost empty of contacts except for two isolated pairs, that are very similar in the two phases. A distance range of interaction energy continuum begins at $R>10 \AA$, less and less directional and evolving towards a point-like ion-ion interaction mode. This is exactly what it is expected in organic salts. ${ }^{14}$

\subsection{Molecular rotations in the crystal}

Some simple computer experiments probe of the ease of molecular reorientation for cations, even in a rigid lattice. A crystal slab made of 192 anion-cation pairs was prepared from the crystal structure of the alpha phase at $278 \mathrm{~K}$ by $4 \times 4 \times 3$ repetitions of the unit cell. A Monte Carlo procedure involving rigid molecules, whose position is described by center-of-mass vectors and three angles of orientation in the inertial molecular reference system was then applied. ${ }^{31,32}$

In a first experiment, one cation molecule at the center of the crystal box is manually reoriented from 0 to $180^{\circ}$ in steps of $30^{\circ}$ around the main inertial axis, roughly coinciding with the normal to the mean molecular plane of the cation (Figure 6 shows the effect of the $180^{\circ}$ reorientation on a sample box without anions). The lattice energy is then calculated for the crystal including the central defect by atom-atom intermolecular potentials. When no relaxation is allowed, an intermolecular energy barrier 
of $9 \mathrm{~kJ} \mathrm{~mol}^{-1}$ develops around $90^{\circ}$ rotation, but if a short $10 \mathrm{kstep} \mathrm{MC} \mathrm{relaxation} \mathrm{is} \mathrm{allowed,} \mathrm{the} \mathrm{barrier}$ vanishes. This demonstrates that the globular cations can reorient quite easily even within an almost stationary lattice.

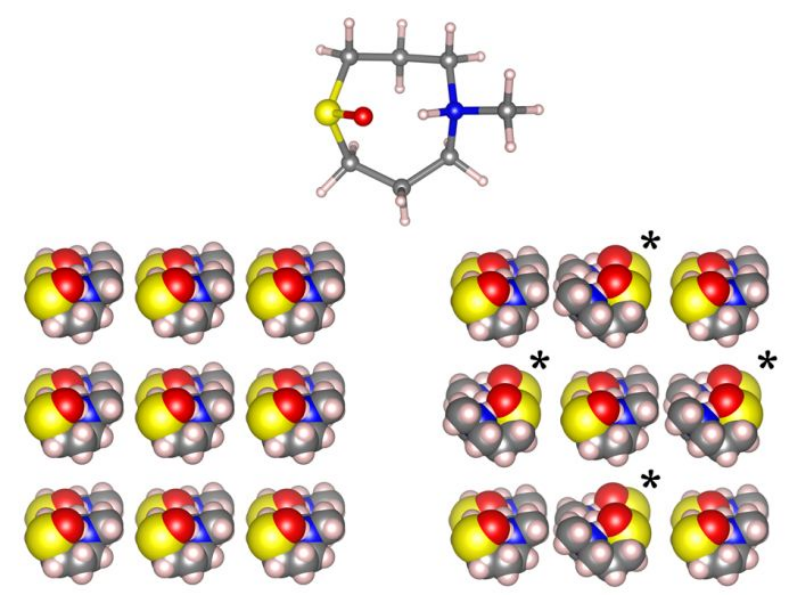

Figure 6. (a) Rows of cations in the ordered crystal. (b) A computer-generated picture in which every second pair in each row (starred) is rotated by $180^{\circ}$ around the axis perpendicular to the average ring plane (use the $\mathrm{S}-$ yellow to $\mathrm{O}-$ red vector as a pointer). The rotation is well described by the angle between transannular vectors from S (yellow) to N (blue): see also Figure 7.

The same conclusion can be reached by just inspection of the cluster geometries, which show no hard contacts at whatever reorientation step. In a second experiment, the 192-pairs slab is manually seeded by $90^{\circ}$ reorientation of every third cation. The same $10 \mathrm{kstep} \mathrm{MC}$ relaxation is applied, again reducing the energy rise to a handful of $\mathrm{kJ} \cdot \mathrm{mol}^{-1}$. Figure 7 shows the distribution of the angles between transannular, S-N intramolecular vectors (Figure 1b,c) after relaxation: significant population of $90^{\circ}$ rotated molecules remains with practically zero energy cost. These simulations do not allow for ring flipping, but it is unlikely that this small conformational rearrangement may change substantially the energetic picture for overall ring tumbling. 


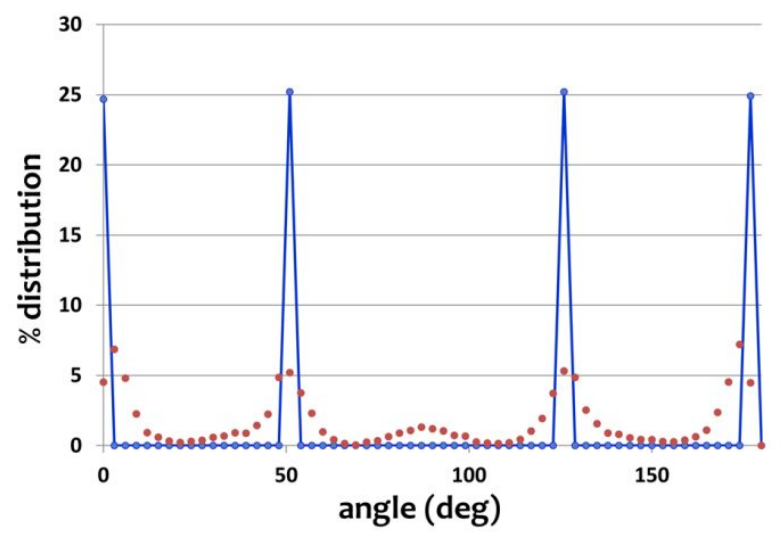

Figure 7. Percent distribution of angles between S-N intramolecular vectors (see text) in the 192-pairs crystal cluster. Blue: ordered crystal, four $25 \%$ peaks corresponding to the four symmetry operations of the space group; red: cluster seeded by $90^{\circ}$ rotation of the cations and then briefly relaxed in Monte Carlo for 10 ksteps. A significant population of $90^{\circ}$ rotated cations remains but the total intermolecular energy of the two distributions differ only by a handful of $\mathrm{kJ} \cdot \mathrm{mol}^{-1}$.

\subsection{Ring flipping}

The possibility of cation ring pseudorotation is crucial to subsequent discussions of the transition mechanism. This point therefore requires more consideration by a survey of available experimental and theoretical evidence.

a) Static evidence. Cyclooctane itself shows a total of 10 different conformations (see Figure S2 ESI) ${ }^{33}$ although MM, DFT and ab initio MO calculations found that just four conformers are stable and populated in the gas phase (boat-chair, BC; twist boat-chair, TBC; twist chair-chair, TCC and crown, CR). ${ }^{34}$ The other forms (boat-boat, BB; twist-boat, TB; twist chair, TC and chair-chair, CC) represent either unstable or high-energy conformations. Chair (C) and boat (B) conformers correspond to transition states. The conformational adaptability of a cyclooctane ring in crystals has been studied by $\mathrm{B} 3 \mathrm{LYP} / 6-31+\mathrm{G}^{*}$ theoretical calculations, ${ }^{33}$ finding energy differences of the order of $1-10 \mathrm{~kJ} \mathrm{~mol}^{-1}$. The $\mathrm{BC}-$ Crown energy difference has been estimated at $6 \mathrm{~kJ} \mathrm{~mol}^{-1}$ by high level quantum chemistry calculations. $^{34}$ All conformers can be divided in four easily interconvertible families. ${ }^{35,36} \mathrm{~A}$ conformational exchange occurs by pseudorotation within a family but only by inversion among families with a higher energy barrier. ${ }^{36}$ The theoretical results agree with crystallographic evidence in confirming that $\mathrm{BC}$ is the dominant conformer. Statistical analysis of other classes of compounds, including substituted carbocycles, C-ring with endo- or exo-double bonds, heterocycles, etc. reveals that conformational preferences in the solid state are the same for chemically modified 8-ring systems, 
although with some deformations. ${ }^{37}$ Excluding bridged or very complex ring systems, deviation from the BC conformer was mostly observed in extended fused-rings or for unsaturated and multiple heterosubstituted rings. Needless to say, crystal packing effects can affect the relative stability of the conformers in the crystal structures.

b) Dynamic evidence. Solid-state conformational changes in ring systems have been characterized by NMR, spectroscopic and crystallographic methods. A rapid conformational exchange has been detected by NMR in pefluorocyclo-octane in solution at room $T .^{38}$ Orientational and conformational disorder in a liquid-like cyclooctane included in thiourea crystals has been reported, ${ }^{39}$ while the dynamic nature of the ring flip (half-ring inversion from BB to TCC conformation) in ammonium salts of nefopam has been characterized by CPMAS-NMR and variable-T diffraction measurements. ${ }^{40}$

c) Computational evidence. Our in vacuo quantum simulations (Figure 8) confirm that the two resulting enantiomeric boat-chair conformations are perfectly equivalent, with electronic energies differing by less than $1.0 \mathrm{~kJ} \cdot \mathrm{mol}^{-1}$ (Table S2 ESI). Accordingly, the pseudorotation path is also symmetric and crosses two slightly distorted, mirror-symmetric equivalent high-energy crown conformers CR and CR' (Figure 8), which lie at $\sim 12 \mathrm{~kJ} \cdot \mathrm{mol}^{-1}$ above the BC/CB states. Thus, M06/6$311 \mathrm{G}(\mathrm{p}, \mathrm{d})$ calculations predict two symmetry-independent elementary steps for the $\mathrm{BC} / \mathrm{CB}$ interconversion, involving both the CR and CR' high-energy minima (Figure 8, Table S2 ESI):

$$
\mathrm{BC} \leftrightarrow \mathrm{TS} 1 \leftrightarrow \mathrm{CR} \leftrightarrow \mathrm{TS} 2 \leftrightarrow \mathrm{CR}^{\prime} \leftrightarrow \mathrm{TS} 3 \leftrightarrow \mathrm{CB}
$$

The equivalent TS1 and TS3 saddle points involve a rather substantial barrier of $\sim 45 \mathrm{~kJ} \cdot \mathrm{mol}^{-1}$ for the $\mathrm{BC} \leftrightarrow \mathrm{CR}$ conversion. The second barrier ( $\mathrm{CR} \leftrightarrow \mathrm{CR}^{\prime}$ ) amounts to $\sim 3.3 \mathrm{~kJ} \cdot \mathrm{mol}^{-1}$, meaning that, once either CR state is achieved, a very fast switching between the two allowed crown conformations must occur. The whole $\mathrm{BC} \leftrightarrow \mathrm{CB}$ conformational rearrangement implies a significant distortion of the $\left(\mathrm{CH}_{2}\right)_{3}-$ carbon ring groups involved in the flipping movement, with changes in covalent bond lengths and angles up to $0.05 \AA$ and $11 \mathrm{deg}$ respectively (Figure S3, Table S3 ESI). Likely, these distortions are responsible for the high kinetic barriers associated to transition states TS1 and TS3 (Figure 8), as the latter imply longer (weakened) $\mathrm{C}-\mathrm{C}$ bonds, in conjunction with large deviations from ideal $\mathrm{sp}^{3}$ geometries of the methylene groups. Also steric effects might play a role, as in TS1 and TS3 hydrogen atoms of adjacent $\mathrm{CH}_{2}$ moieties are forced into an almost eclipsed arrangements. Interestingly, the geometry of the transannular $\mathrm{NH} \cdots \mathrm{O}$ hydrogen bond is poorly affected: the $\mathrm{H} \cdots \mathrm{O}$ distance undergoes a 
slight average decrease, from $1.57 \AA$ to $1.56 \AA$, on going from either the $\mathrm{BC}$ or the $\mathrm{CB}$ state to the closest transition saddle point, TS1 or TS3. Then, it is lengthened, on average by $\sim 0.06 \AA$, when the ring achieves either the $\mathrm{CR}$ or CR' crown conformation (Figure S3a ESI). The corresponding maximum distortions of the S-H-O angle, however, are smaller than $3.5 \mathrm{deg}$ (Table S3 ESI). This suggests that the transannular hydrogen bond is strong enough to be conserved throughout the conformational rearrangement. Therefore, it is reasonable to assume that it prevents TACO from exploiting the full conformational flexibility observed in cyclooctane, contributing to increase the kinetic barrier of the pseudorotation process.

Some experimental evidence suggests that conformational interchanges of medium-size rings probably occur by stepwise mechanisms rather than via synchronized changes involving the whole cycle. $^{40-42}$ Our gas-phase findings agree with such a model: the ring inversion in TACO is achieved through a two-step mechanism, each of which implies the inversion of a half of the cycle at a time. The latter is obtained through a step-by-step displacement of a pair of $-\mathrm{CH}_{2}$ - groups across the main ring plane. ${ }^{43,44}$ Moreover, the high-energy minima CR and CR' could account for the possible occurring of a metastable crystal structure somewhat intermediate between the $\alpha$ and $\beta$ polymorphs, whose existence was claimed in the past by Paul and co-workers. ${ }^{12}$ However, considering the kinetics of the whole process (see Figure 8 and Conclusions below), such a structure, if exists, should be just a transient one, and we expect that it could be hardly isolated.

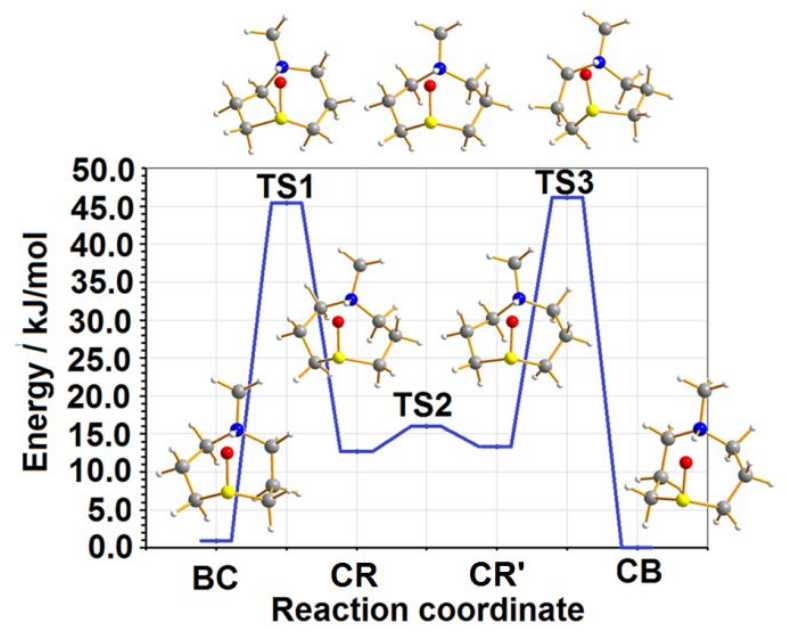

Figure 8. The quantum M06/6-311G(p,d) energy profile for a possible interconversion of the TACO cation ring system.

\section{Conclusions}


Our computational analysis has pinpointed a number of relevant facts in the polymorphic equilibrium of TACO, revealing the subtle energy differences that characterize the two phases. However, one is now faced with the key question: what is the molecular-level mechanism of the TACO polymorphic transformation?

At one extreme of the range of possibilities, the phase change may occur by complete disruption of the crystal structure of the mother phase into a bulk liquid, possibly starting at defects, and successive recrystallization of the daughter phase. In such a scenario, the phase change can be regarded as a sort of pre-melting feature. Our results show that molecular rotations at or about the temperature of the phase transition are relatively easy, the anion being almost perfectly spherical and the cation being also close to a globular shape. Translational diffusion may also be relatively unhampered for the same reasons. However, some experimental evidence stands against this picture for the TACO case: there is no sign of Bragg diffraction breakdown near the transformation temperature; and the transformation is easily reversible. ${ }^{11-13}$

At the other extreme of the transformation landscape, much more attractive, if still puzzling, would be a thoroughly on-site process, as suggested by Figures 2, 3 and 6, involving in every second cation molecule only minor displacements of the centers of mass, with $180^{\circ}$ molecular rotation, along with a conformational flip of the boat-chair ring with chirality switch, and with anions following suit. Several pieces of our computational evidence have a say on this proposed model.

a) Although the difference in total lattice energy between polymorphs is very small, partitioned molecule-molecule energies show that the rearrangement involves a number of surprising retentions of interaction, but also that quite a few molecule-molecule contacts undergo a substantial energetic change in the local environment, as seen in Table 3 and Figure 5a. Therefore the picture shown in Figure 2 apparently suggesting an almost superimposable pattern of the two structures, is in good part misleading, as usually are inferences based only on geometry without supporting quantitative evidence.

b) Molecular simulation shows that the globular cations can undergo rotational or translational diffusion with only minor energy penalty. The packing of nearly spherical anions should be scarcely structure- or orientation-selective (Figures 2-4), and such spherical shapes should also be scarcely selective against anion-cation interaction. Molecular mobility must be rather high and in this respect the on-site phase change mechanism seems accessible. Partly countering this conclusion is the fact that no unusual behavior of atomic displacement parameters from Xray diffraction seems to occur near the transition temperature. 
c) The sulfinyl $(\mathrm{S}=\mathrm{O})$ group forms a strong intramolecular 1,5-transannular hydrogen bond with the protonated nitrogen atom (Figure 1), making the molecule almost a bridged species in what seems to be a hinge effect against full conformational flexibility. This effect stabilizes both BC and crown conformers, but as a result, quantum calculations show that flipping of the eight-membered ring is hampered by a rather substantial barrier of $45 \mathrm{~kJ} \mathrm{~mol}^{-1}$, high enough against $3 R T$ to quench a pseudorotation. The result refers to a gas-phase molecule, but an assist from the intermolecular field in lowering this barrier seems rather unlikely.

In conclusion, the present computational evidences imply that a site-by-site mechanism, where conformational changes of cations occur in conjunction with very easy rotations of both the ions, seems to be preferred. Even though from a thermodynamic viewpoint the $\alpha$ and $\beta$ polymorphs of TACO are nearly isoenergetic (Table 2), the phase transition implies a substantial redistribution of the moleculemolecule interaction energies (Figure 5, Table 3), where both Coulomb repulsive and attractive terms undergo changes up to several hundreds of $\mathrm{kJ} \cdot \mathrm{mol}^{-1}$. In this scenario, it seems quite reasonable that the kinetic cost of the $\mathrm{BC} \rightarrow \mathrm{CR}$ conformational change across the $\beta \rightarrow \alpha$ transition could be paid by relieving some Coulomb repulsions among neighboring cations. Then, an almost identical amount of energy is released upon the subsequent crown/boat-chair conversion (Figure 8) and it eventually does not appear in the overall thermodynamic balance. Upon warming, the structural change can be inverted once the energy necessary to overcome the inverse kinetic barrier is provided by an external source. This picture is in agreement with the fact that the low- $T$ structure $\alpha$ is not stable above the transition temperature, but the high- $T$ phase $\beta$ can be supercooled into a metastable regime. It also complies well with the preservation of the macroscopic crystal shape and the permanence of Bragg diffraction across the first-order phase transition. At the same time, this scenario corroborates the idea that TACO does not conform to classical topochemical postulates - that is, the $\beta / \alpha$ transition does not occur through small atomic and molecular movements. Rather, the crystal can be considered as a sort of "motor at molecular level", as it uses either its internal energy $(\beta \rightarrow \alpha)$ or the heat provided from an external source $(\alpha \rightarrow \beta)$ to produce work in terms of a large, non-trivial and fully reversible internal coherent motion of atoms.

\section{Acknowledgements.}


This research was partially funded by the Unimi Development Plan-Line 2, Action B, project NOVAQ, nº PSR2015-1716FDEMA_08.

\section{Supporting Information Available.}

Crystal density of $\alpha-$ and $\beta-$ TACO as a function of temperature. Full comparison of center-of-mass distances and molecule-molecule interaction energies. Canonical conformations of cyclooctane. Electronic energies of TACO conformers and transition states in the gas phase, with selected bond lengths and angles.

\section{References}

(1) Dunitz, J. D. Phase Transitions in Molecular Crystals: Looking Backwards, Glancing Sideways. Phys. Scr. 2016, 91 (11), 112501.

(2) Mnyukh, Y. V.; Panfilova, N. A.; Petropavlov, N. N.; Uchvatova, N. S. Polymorphic Transitions in Molecular Crystals-III. J. Phys. Chem. Solids 1975, 36 (3), 127-144.

(3) van den Ende, J. A.; Ensing, B.; Cuppen, H. M. Energy Barriers and Mechanisms in Solid-solid Polymorphic Transitions Exhibiting Cooperative Motion. CrystEngComm 2016, 18 (23), 44204430 .

(4) Naumov, P.; Bharadwaj, P. K. Single-Crystal-to-Single-Crystal Transformations. CrystEngComm. 2015.

(5) Brittain, H. G.; Morris, K. R.; Bugay, D. E.; Thakur, A. B.; Serajuddin, A. T. M. Solid-State NMR and IR for the Analysis of Pharmaceutical Solids: Polymorphs of Fosinopril Sodium. $J$. Pharm. Biomed. Anal. 1993, 11 (11-12), 1063-1069.

(6) Baikie, T.; Fang, Y.; Kadro, J. M.; Schreyer, M.; Wei, F.; Mhaisalkar, S. G.; Graetzel, M.; White, T. J. Synthesis and Crystal Chemistry of the Hybrid Perovskite (CH3NH3)PbI3 for Solid-State Sensitised Solar Cell Applications. J. Mater. Chem. A 2013, 1 (18), 5628.

(7) Destro, R.; Ortoleva, E.; Soave, R.; Loconte, L.; Lo Presti, L. Detection and Kinetics of the Single-Crystal to Single-Crystal Complete Transformation of a Thiiranium Ion into Thietanium Ion. Phys. Chem. Chem. Phys. 2009, 11 (33), 7181.

(8) Destro, R.; Sartirana, E.; Loconte, L.; Soave, R.; Colombo, P.; Destro, C.; Lo Presti, L. Competing $\mathrm{C}=\mathrm{O} \cdots \mathrm{C}=\mathrm{O}, \mathrm{C}-\mathrm{H} \cdots \mathrm{O}, \mathrm{Cl} \cdots \mathrm{O}$, and $\mathrm{Cl} \cdots \mathrm{Cl}$ Interactions Governing the Structural 
Phase Transition of 2,6-Dichloro-p- Benzoquinone at $\mathrm{T}_{\mathrm{c}}=122.6 \mathrm{~K}$. Cryst. Growth Des. 2013, 13 (10), 4571-4582.

(9) Berry, D. J.; Seaton, C. C.; Clegg, W.; Harrington, R. W.; Coles, S. J.; Horton, P. N.; Hursthouse, M. B.; Storey, R.; Jones, W.; Friščić, T.; et al. Applying Hot-Stage Microscopy to Co-Crystal Screening: A Study of Nicotinamide with Seven Active Pharmaceutical Ingredients. Cryst. Growth Des. 2008, 8 (5), 1697-1712.

(10) Wishkerman, S.; Bernstein, J. Polymorphism and Structural Mechanism of the Phase Transformation of Phenyl Carbamate (PC). Chem. - A Eur. J. 2008, 14 (1), 197-203.

(11) Paul, I. C.; Go, K. T. The Crystal and Molecular Structure of 5-Methyl-1-Thia-5-AzacycloOctane 1-Oxide Perchlorate. J. Chem. Soc. B Phys. Org. 1969, No. 0, 33.

(12) Duesler, E. N.; Wiegers, K. E.; Curtin, D. Y.; Paul, I. C. Experimental Studies on the Single Crystal to Single Crystal Transformation of 5-Methyl-1-Thia-5-Azacyclooctane-1-Oxide (TACO) Perchlorate. Evidence for an Intermediate State. Mol. Cryst. Liq. Cryst. 1980, 59 (3-4), 289-298.

(13) Novotny-Bregger, E. Beobachtungen von Phasenübergängen Bei Einigen Polymorphen Organischen Verbindungen; ETH-Zurich, D. D., Ed.; Doctoral Dissertation ETH-Zurich, 1994.

(14) Macetti, G.; Loconte, L.; Rizzato, S.; Gatti, C.; Lo Presti, L. Intermolecular Recognition of the Antimalarial Drug Chloroquine: A Quantum Theory of Atoms in Molecules-Density Functional Theory Investigation of the Hydrated Dihydrogen Phosphate Salt from the 103 K X-Ray Structure. Cryst. Growth Des. 2016, 16 (10), 6043-6054.

(15) Parkinson, G. M.; Thomas, J. M.; Williams, J. O.; Goringe, M. J.; Hobbs, L. W. The Suggested Role of Partial Dislocations in the Single Crystal $\rightleftarrows$ Single Crystal Phase Transition of a CycloOctane Molecular Cationic Salt. J. Chem. Soc. Perkin Trans. 2 1976, No. 7, 836-838.

(16) Mei, Z.; Zhao, F.-Q.; Xu, S.-Y.; Ju, X.-H. Theoretical Investigations on the Phase Transition of Pure and Li-Doped AlH 3. RSC Adv. 2017, 7 (67), 42024-42029.

Achary, S. N.; Errandonea, D.; Muñoz, A.; Rodríguez-Hernández, P.; Manjón, F. J.; Krishna, P. S. R.; Patwe, S. J.; Grover, V.; Tyagi, A. K. Experimental and Theoretical Investigations on the Polymorphism and Metastability of $\mathrm{BiPO}_{4}$. Dalt. Trans. 2013, 42 (42), 14999.

(18) Ectors, P.; Sae-Tang, W.; Chatchawalsaisin, J.; Zahn, D.; Anwar, J. The Molecular Mechanism of $\alpha$-Resorcinol's Asymmetric Crystal Growth from the Melt. Cryst. Growth Des. 2015, 15 (8), 
4026-4031.

(19) Maschio, L.; Civalleri, B.; Ugliengo, P.; Gavezzotti, A. Intermolecular Interaction Energies in Molecular Crystals: Comparison and Agreement of Localized Møller-Plesset 2, DispersionCorrected Density Functional, and Classical Empirical Two-Body Calculations. J. Phys. Chem. A 2011, 115 (41), 11179-11186.

(20) Dunitz, J. D.; Gavezzotti, A. Molecular Recognition in Organic Crystals: Directed Intermolecular Bonds or Nonlocalized Bonding? Angew. Chemie Int. Ed. 2005, 44 (12), 17661787.

(21) Groom, C. R.; Allen, F. H. The Cambridge Structural Database in Retrospect and Prospect. Angew. Chemie - Int. Ed. 2014, 53 (3), 662-671.

(22) Allen, F. H.; Bruno, I. J. Bond Lengths in Organic and Metal-Organic Compounds Revisited: X - H Bond Lengths from Neutron Diffraction Data. Acta Crystallogr. Sect. B Struct. Sci. 2010, 66 (3), 380-386.

(23) Gavezzotti, A. Efficient Computer Modeling of Organic Materials. the Atom-Atom, CoulombLondon-Pauli (AA-CLP) Model for Intermolecular Electrostatic- Polarization, Dispersion and Repulsion Energies. New J. Chem. 2011, 35 (7), 1360-1368.

(24) Frisch, M. J. . et al. Official Gaussian 09 Literature Citation. Gaussian 09, Revis. D.01; Gaussian Inc. Wallingford CT J. 2009.

(25) Fukui, K. The Path of Chemical Reactions - The IRC Approach. Acc. Chem. Res. 1981, 14 (12), $363-368$.

(26) Zhao, Y.; Truhlar, D. G. The M06 Suite of Density Functionals for Main Group Thermochemistry, Thermochemical Kinetics, Noncovalent Interactions, Excited States, and Transition Elements: Two New Functionals and Systematic Testing of Four M06-Class Functionals and 12 Other Function. Theor. Chem. Acc. 2008, 120 (1-3), 215-241.

(27) Colombo, V.; Lo Presti, L.; Gavezzotti, A. Two-Component Organic Crystals without Hydrogen Bonding: Structure and Intermolecular Interactions in Bimolecular Stacking. CrystEngComm 2017, 19 (17), 2413-2423.

(28) Gavezzotti, A.; Colombo, V.; Lo Presti, L. Facts and Factors in the Formation and Stability of Binary Crystals. Cryst. Growth Des. 2016, 16 (10), 6095-6104.

(29) Gavezzotti, A.; Lo Presti, L. Building Blocks of Crystal Engineering: A Large-Database Study of the Intermolecular Approach between C-H Donor Groups and O, N, Cl, or F Acceptors in 
Organic Crystals. Cryst. Growth Des. 2016, 16 (5), 2952-2962.

(30) Gavezzotti, A.; Lo Presti, L. Theoretical Study of Chiral Carboxylic Acids. Structural and Energetic Aspects of Crystalline and Liquid States. Cryst. Growth Des. 2015, 15 (8), 37923803.

(31) Gavezzotti, A. Comparing the Strength of Covalent Bonds, Intermolecular Hydrogen Bonds and Other Intermolecular Interactions for Organic Molecules: X-Ray Diffraction Data and Quantum Chemical Calculations. New J. Chem. 2016, 40 (8), 6848-6853.

(32) Gavezzotti, A. Equilibrium Structure and Dynamics of Organic Crystals by Monte Carlo Simulation: Critical Assessment of Force Fields and Comparison with Static Packing Analysis. New J. Chem. 2013, 37 (7), 2110-2119.

(33) Eliel, E. L.; Wilen, S. H. Stereochemistry of Organic Compounds; Wiley India Pvt. Limited, 2008.

(34) Wiberg, K. B. The C7-C10 Cycloalkanes Revisited. J. Org. Chem. 2003, 68 (24), 9322-9329.

(35) Hendrickson, J. B. Molecular Geometry. V. Evaluation of Functions and Conformations of Medium Rings. J. Am. Chem. Soc. 1967, 89 (26), 7036-7043.

(36) Dragojlovic, V. Conformational Analysis of Cycloalkanes. ChemTexts 2015, 1 (3), 14.

(37) Allen, F. H.; Howard, J. A. K.; Pitchford, N. A. Symmetry-Modified Conformational Mapping and Classification of the Medium Rings from Crystallographic Data. IV. Cyclooctane and Related Eight-Membered Rings. Acta Crystallogr. Sect. B Struct. Sci. 1996, 52 (5), 882-891.

(38) Anderson, J. E.; Glazer, E. S.; Griffith, D. L.; Knorr, R.; Roberts, J. D. Nuclear Magnetic Resonance Spectroscopy. Conformations and Conformational Equilibration of Some Cyclooctane Derivatives. J. Am. Chem. Soc. 1969, 91 (6), 1386-1395.

(39) Maris, T.; Henson, M. J.; Heyes, S. J.; Prout, K. Investigations of the Phase Transitions in Thiourea Inclusion Compounds with Cycloheptane, Cyclooctane, and Cyclooctanone. Chem. Mater. 2001, 13 (8), 2483-2492.

(40) Glaser, R.; Novoselsky, A.; Shiftan, D.; Drouin, M. Eight-Membered-Ring Solid-State Conformational Interconversion via the Atom-Flip Mechanism, a CP-MAS 13C NMR and Crystallographic Stereochemical Study. J. Org. Chem. 2000, 65 (20), 6345-6353.

(41) Glaser, R.; Shiftan, D.; Levi-Roso, G.; Ergaz, I.; Geresh, S.; Drouin, M. Cis-Cyclononene Conformational Families and a Crystallographic Example of a Skew-Chair-Boat Type-2 Conformation. J. Org. Chem. 2002, 67 (16), 5486-5496. 
(42) Glaser, R.; García, A.; Chávez, M. I.; Delgado, G. The Solid-State and Solution-State Reassigned Structures of Tagitinin A, a 3,10-Epoxy-Germacrolide from Tithonia Diversifolia, and the Interconversion of 3,10-Epoxy-Germacrolide Conformational Families via a Ring-Atom Flip Mechanism. J. Braz. Chem. Soc. 2005, 16, 440-448.

(43) Glaser, R. Symmetry, Spectroscopy, and Crystallography: The Structural Nexus; Wiley, 2015.

(44) Goto, H.; Osawa, E. Corner Flapping: A Simple and Fast Algorithm for Exhaustive Generation of Ring Conformations. J. Am. Chem. Soc. 1989, 111 (24), 8950-8951. 


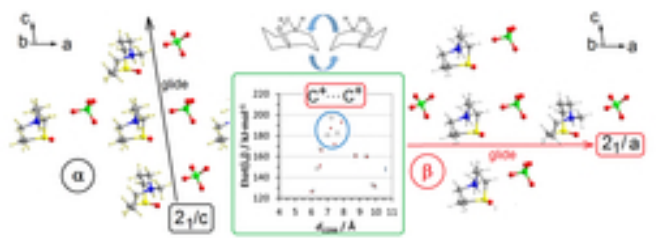

Synopsis: The energy decomposition analysis reveals that the elusive $a \leftrightarrow \beta$ phase transition of TACO should imply a fast rotation of both anions and cations, coupled to a slow boat-chair $\leftrightarrow$ chair-boat interconversion of the 8-membred ring.

$21 \times 7 \mathrm{~mm}(300 \times 300 \mathrm{DPI})$ 


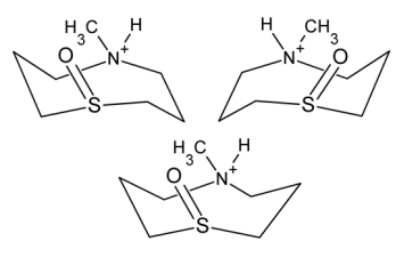

a)

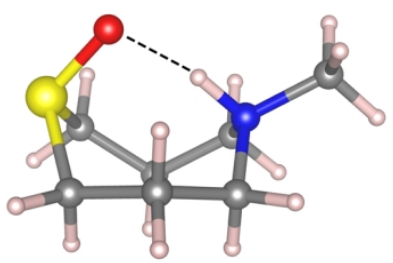

b)

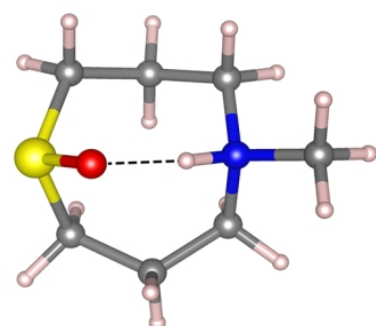

c)

The TACO cation molecule: a) two enantiomers in the boat-chair conformation, and a hypothetical crown conformation; b) and c), two views of the crystalline cation, showing the transannular intramolecular $\mathrm{S}=\mathrm{O} \cdots \mathrm{H}-\mathrm{N}$ hydrogen bond. c) a view down the main inertial axis. The perchlorate anion has a regular tetrahedral shape.

$150 \times 45 \mathrm{~mm}(300 \times 300 \mathrm{DPI})$ 
Left: a-phase at $278 \mathrm{~K}$ : translation along a, horizontal row; glide along c, vertical row. Right: $\beta$-phase at 298K: glide along a, horizontal row; translation along C, vertical row. Symmetry operations and crystallographic reference frames are shown. Numerals serve to unequivocally label pairs of molecules whose contact energies are discussed (see infra); primed numbers refer to translation-related pairs. The $b$ axis is tilted by approximately $\sim 14^{\circ}$ with respect the plane normal of the picture.

$150 \times 53 \mathrm{~mm}(300 \times 300 \mathrm{DPI})$ 
(a) a-phase, translation ribbon along a. Cation molecules obviously preserve orientation and chirality. (b) $\beta-$ phase, the glide reflection along a $(x, y, z ; x+1 / 2,-y-1 / 2, z)$ (blue arrow) and the "equivalent" rotationflipping operation (pink arrow) showing that molecule $B$ is transformed with respect to $A$ by a $180^{\circ}$ rotation around an axis approximately perpendicular to the mean ring plane, along with a boat-chair to chair-boat ring flip (hydrogens omitted for clarity).

$$
94 \times 71 \mathrm{~mm}(300 \times 300 \mathrm{DPI})
$$




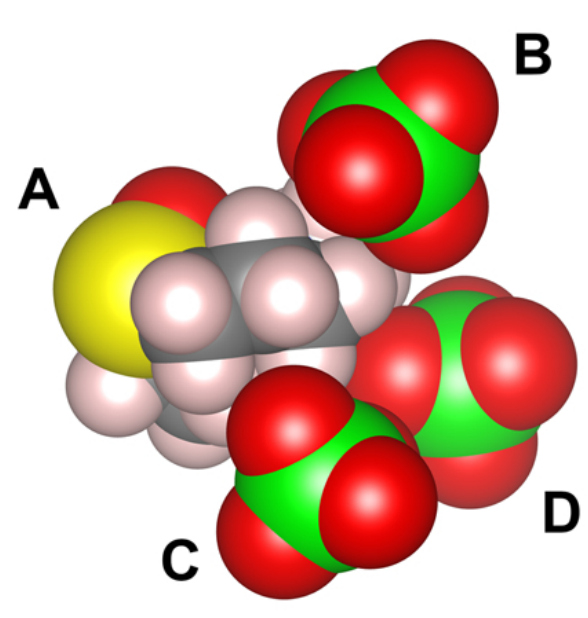

a-phase

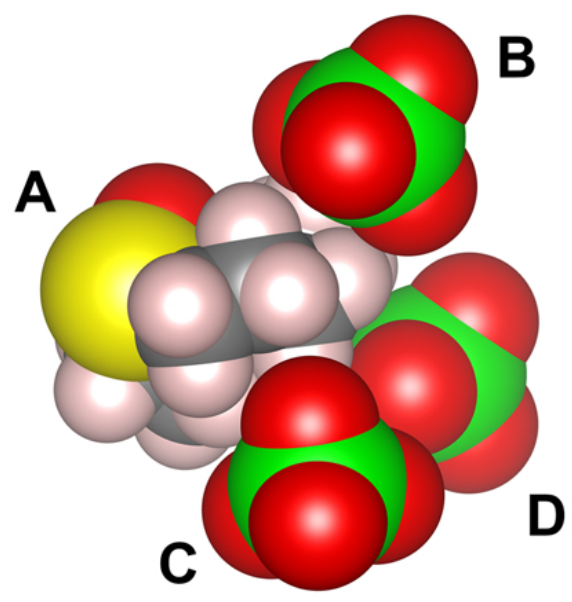

$\beta$-phase

The cation ( $A$ ) and its three closest-interacting anions (B, C, D). Negative oxygens tend to cluster around positive methylene hydrogen regions in both phases. The A-B contact is almost identical in the two phases.

$80 \times 46 \mathrm{~mm}(300 \times 300 \mathrm{DPI})$ 


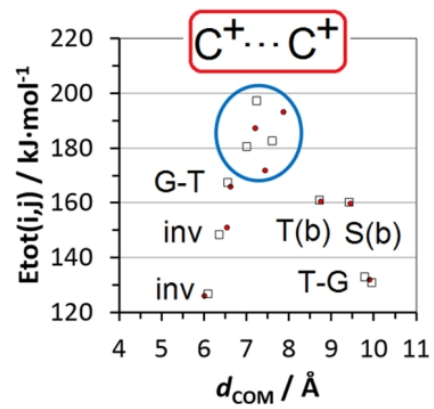

(a)

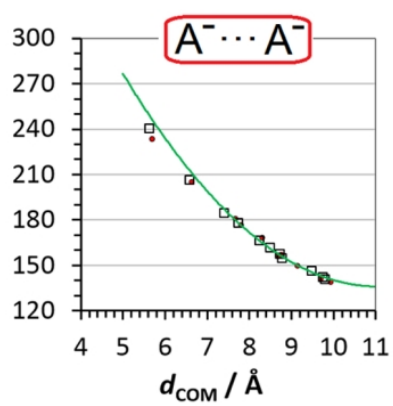

(b)

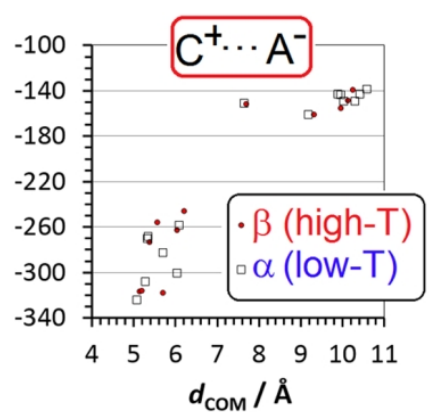

(c)

(a) Distance-energy pattern for the cation-cation contacts in the two phases. $d$ com is the centre-of-mass distance. Identical pairs: two inversion-related (inv) plus one b-translation ( $T(b)$ ) and one screw pair (S(b)). G-T and T-G are the pairs interchanged between translation and glide in the ac plane. The blue circle encloses pairs where $d_{\text {COM }}$ changes by about $0.3 \AA$ and $E_{\text {tot }}(i, j)$ by about $10 \mathrm{~kJ} \mathrm{~mol}^{-1}$ on changing phase. (b) Same as (a), for the anion-anion contacts. The full green line follows the Coulomb law for a couple of ions with identical charges, as a function of $d_{\mathrm{COM}}$. (b) Same as (a), for the cation-anion contacts.

$150 \times 58 \mathrm{~mm}(300 \times 300$ DPI $)$ 
(a) Rows of cations in the ordered crystal. (b) A computer-generated picture in which every second pair in each row (starred) is rotated by $180^{\circ}$ around the axis perpendicular to the average ring plane (use the Syellow to O-red vector as a pointer). The rotation is well described by the angle between transannular vectors from $\mathrm{S}$ (yellow) to $\mathrm{N}$ (blue): see also Figure 7.

$80 \times 60 \mathrm{~mm}(300 \times 300 \mathrm{DPI})$ 
Percent distribution of angles between S-N intramolecular vectors (see text) in the 192-pairs crystal cluster. Blue: ordered crystal, four $25 \%$ peaks corresponding to the four symmetry operations of the space group; red: cluster seeded by $90^{\circ}$ rotation of the cations and then briefly relaxed in Monte Carlo for 10 ksteps. A significant population of $90^{\circ}$ rotated cations remains but the total intermolecular energy of the two distributions differ only by a handful of $\mathrm{kJ} \cdot \mathrm{mol}^{-1}$.

$80 \times 55 \mathrm{~mm}(300 \times 300$ DPI $)$ 


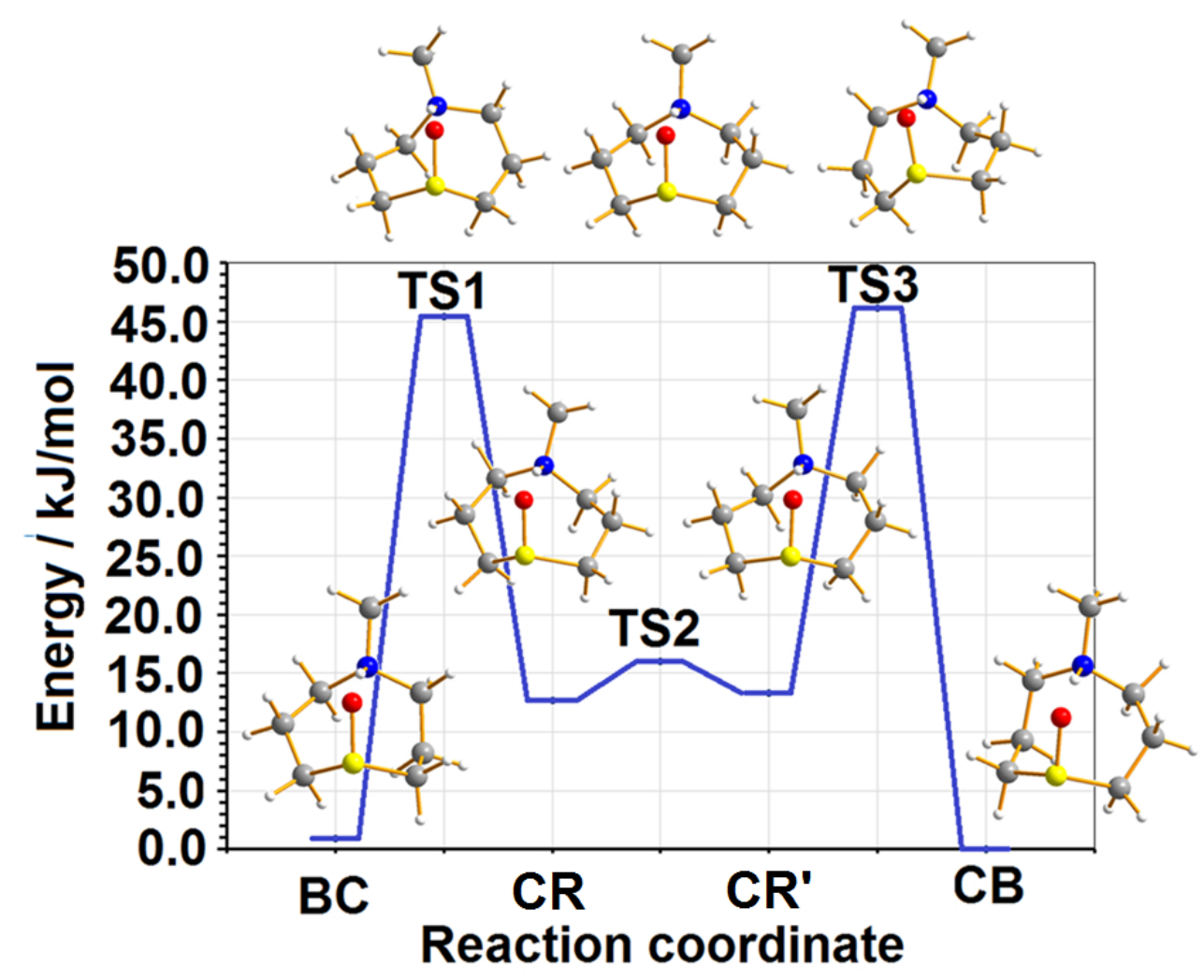

The quantum M06/6-311G(p,d) energy profile for a possible interconversion of the TACO cation ring system.

$80 \times 65 \mathrm{~mm}(300 \times 300$ DPI $)$ 